\title{
Improved Contrast Infrared Small Target Detection Algorithm Based on Local Edge Extraction
}

\author{
Shuai Wang ${ }^{1}$, Zaiping Lin'1, Hongwei Cheng² \\ ${ }^{1}$ College of Electronic Science and Engineering, National University of Defense Technology, \\ Changsha, Hunan, China \\ ²Beijing Institute of Tracking and Telecommunication Technology, Beijing 100094, China
}

Keywords: dim and small target, visual saliency, local contrast, canny operator

\begin{abstract}
An algorithm of small and dim infrared target detection base on LCM (Local Contrast Method) combing with edge detection to solve the problem of high false alarm in small and dim infrared target detection under complex background. This algorithm firstly uses the LCM (Local Contrast Method) to extract the visual salient region in the image. Then the Canny operator is used to extract the edge of the cloud. The next we will get the local contrast map to compare with the result of Canny operator algorithm, the target which is located of the cloud edge will be eliminated. The last, adopt to threshold segmentation to separate the target. Analysis of the result shows that proposed algorithm which is based on LCM and Canny operator has a better detection result in small and dim infrared target detection under cloudy background. What's more, it can solve the problem of the high false alarm rate by LCM algorithm.
\end{abstract}

\section{Introduction}

Infrared surveillance warning system is an important part of modern warfare defense system, which has the characteristics of long monitoring distance, rapid response, etc. It has become an important factor in the military competition among the great powers. Because of the long detection distance, the image of the missile is displayed as a dim and small target in the infrared image. Therefore, the detection of dim and small targets using infrared detection image under complex background conditions is one of the key technologies of infrared missile warning. Due to the existence of the solar light reflection and the cloud background, the infrared detection image SNR is low, the target easily submerged in the background clutter, the detection is very difficult. Therefore, the effective target detection algorithm is very important to improve the performance of monitoring system.

Dim and small targets are not related to the surrounding neighborhood, because they occupy only a small area in the infrared image. The detection process is susceptible to background noise. In human vision, due to the small target energy concentration compared with the surrounding obvious, it can be more visual attention and be considered as the visually sensitive areas. For this feature, researchers have proposed Dog operator, scale space, Log filters and other detection algorithms. These algorithms have achieved good results in the detection of infrared dim and small targets. However, the effect of infrared dim and small target detection in complex background is general. In view of this situation, Chen et. proposed a contrast detection method based on Local Contrast Method(LCM), which uses the human visual system (HVS) characteristics and derived kernel model (DK model), achieved good detection results. In the detection process, it traverses through a local area sliding window of the entire image, calculates the local contrast, to get the local contrast diagram of the whole image. In the local contrast image, the contrast of the cloud clutter region is low, so it is regarded as the background suppression. Target was highlighted due to the high contrast with the background. Then the SNR of the image is enhanced, and finally get the target through threshold segmentation. However, in the detection process, due to the reflection of light clouds, it will enhance the contrast of the cloud edge. The edge of the cloud will be used as a target, resulting in a large number of false alarms. This paper proposes a detection algorithm based on Canny operator to extract 
the false alarm from the cloud edge. The simulation results show that the algorithm can restrain the false alarm of the edge of the cloud, keep the energy of the target, and improve the detection performance.

\section{LCM (local contrast method) algorithm}

Visual attention mechanism plays an important role in the human visual system. It enables us to perceive the valuable information that can cause visual attention in the process of observing things. Thus, visual attention mechanism is widely used in image processing, medicine and psychology, and other fields. In image processing, the visual attention mechanism is applied to the saliency detection of the target, that is, the target is detected in the saliency region.

There are many methods to calculate the saliency map. Chen et. proposed a local contrast method (LCM)algorithm, namely through the sliding window to traversing the entire image, computing each sliding window local contrast, finally get the whole image local contrast map.

The specific calculation steps are as follows:

Step 1: On an image $w$, take the sliding window $v, v$ slide from top to bottom from left to right in the image $w$. Each sliding window is divided into 9 blocks, the number of each area is shown in Fig.1. (b), where the 0 area is the central area $u$.

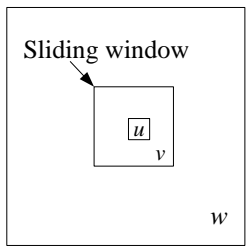

(a)

\begin{tabular}{|l|l|l|}
\hline 1 & 2 & 3 \\
\hline 4 & 0 & 5 \\
\hline 6 & 7 & 8 \\
\hline
\end{tabular}

(b)

Fig. 1. (a) Illustration of the sliding window. (b) Illustration of the image block

Then calculate the mean of each piece, denoted $m_{i}(i=1,2 \ldots 8)$.

$$
m_{i}=\frac{1}{N} \sum_{j=1}^{N} I_{j}^{i}
$$

Step 2: Calculate the local contrast value of the sliding window, define the area around the central area and the contrast values:

$$
c_{i}^{n}=\frac{L_{n}}{m_{i}}
$$

Where $L_{n}$ is the maximum value of the intermediate zone 0 . In order to strengthen the target, the definition of LCM value is:

$$
C_{n}=\min _{i} L_{n} \times c_{i}^{n}=\min _{i} L_{n} \times \frac{L_{n}}{m_{i}}=\min _{i} \frac{L_{n}^{2}}{m_{i}}
$$

In which, $\min _{i} \frac{L_{n}^{2}}{m_{i}}=\frac{L_{n}^{2}}{\max _{i} m_{i}}$. From the formula, we can see that the value of $C_{n}$ and local extreme value $L_{n}$ and $\max _{i} m_{i}$ are related, therefore, this algorithm is easy to be affected by the gray value of individual pixels.

Step 3: Calculate the local contrast of different scales.

Since the pixel size of the target will be changed, and the size of the window $u$ is the same as the target. Therefore, Calculation of LCM values at different size scales is required to calculate the final contrast map $\hat{C}_{p, q} . l_{\max }$ is the maximum size of the target. The contrast map in each scale from 1 to $l_{\text {max }}$ is calculated. And then for the image, the contrast value of each point $\hat{C}_{p, q}$ is:

$$
\hat{C}_{p, q}=\max _{l=1,2, \ldots, l_{\max }} C_{p, q}^{l}
$$

Shortcomings of LCM algorithm. 
Analysis algorithm process can be found, after calculating the local contrast image, the image SNR is significantly improved, the surrounding background is suppressed, and the target can be better detected. However, there are still false alarm rate in the cloud edge. The $L_{n}$ in the formula represents the maximum value in the zeroth pixel block, if a pixel (the target or the noise point, denoted as $I(i, j))$ is the larger value of the gray value. Then all contain $I(i, j)$, the maximum value $L_{n}$ is the same, and the difference among the value of $\max _{i} m_{i}$ in the area of surrounding background is a little.

It will result that the pixel points around $I(i, j)$ will be weighted by the larger $c_{i}^{n}=\frac{L_{n}}{m_{i}}$ the same as $I(i, j)$. And the result is their local contrast value is larger than the normal value, it will be mistaken for target in detection, become false alarm points.

\section{Improved algorithm in this paper}

The edge of the image is one of the basic characteristics of the image, its gray value is not continuous, this characteristic causes it to be easily detected. There are so many kinds of noise in the infrared detection image, more typical is the cloud clutter noise. In the process of target detection, there are a large number of false alarms caused by the edge of the clouds. Therefore, the effective way to eliminate false alarm is to extract the cloud edge, and the edge image is extracted and compared with the local contrast image. The edge extraction algorithm based on Canny operator, due to its strong anti-noise ability and high precision edge detection capability, it is widely used in target detection. That achieved good results for the subsequent reject false alarm Foundation.

\subsection{Canny operator edge extraction algorithm}

Canny convert the edge detection problem to the maximum value of the detection function. Canny operator edge detection process is: first, using the Gauss function convolution image, and then calculate the Gauss function in any direction of the local maxima, the finally obtained is the edge point. The algorithm is as follows:

(1) Gauss filter for the image, that is, using the Gauss function to convolution image, thereby removing the noise in the image.

Two-dimensional Gaussian function as follows:

$$
G(x, y, \sigma)=\frac{1}{2 \pi \sigma^{2}} \exp \left(\frac{1}{2 \sigma^{2}}\left(x^{2}+y^{2}\right)\right)
$$

(2) The Gauss function is calculated as the reciprocal of the first order in any direction, that is, the gradient in the direction.

$$
G_{n}=\frac{\partial G}{\partial n}=\vec{n} \nabla G \quad \vec{n}=\left[\begin{array}{c}
\cos \theta \\
\sin \theta
\end{array}\right]^{T} \quad \nabla G=\left[\begin{array}{c}
\frac{\partial G}{\partial x} \\
\frac{\partial G}{\partial y}
\end{array}\right]
$$

(3) "Non-maximum suppression" of the gradient is to seek the maximum value of the convolution of Gauss's function and image. The point of maximum value is an edge point. Canny algorithm uses a $3 * 3$ array with 8 direction neighborhood to interpolate the gradient magnitude along the gradient direction. The mathematical expression is:

$$
N[i, j]=N M S(M[i, j], Y[i, j])
$$

(4) To connect the edge, Canny operator using the dual-threshold segmentation algorithm to the edge, remove the false edge, and then connected with recursive tracking algorithms to get the edge, finally get the edge to be detected. 


\section{Simulation and Analysis}

In order to verify the effectiveness of the algorithm, this paper will choose LCM (local contrast method) algorithm combined with Canny operator edge detection algorithm to detect weak infrared detection target image. Visual attention mechanism uses local contrast algorithm, the effect of suppressing the outer point of the target background intensity is better, and can enhance the SNR of target point. Because the contrast of the clouds edge is too large, it's easily to generate a large number of false alarms. In this paper, we take the cloud edge detection algorithm based on Canny operator, and compared with the significant map obtained by local contrast algorithm, the false alarm in the edge of the cloud is removed. Next, we first calculate the local contrast image of the infrared image with dim and small targets.

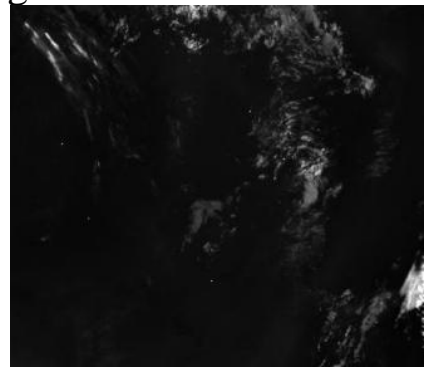

(a)

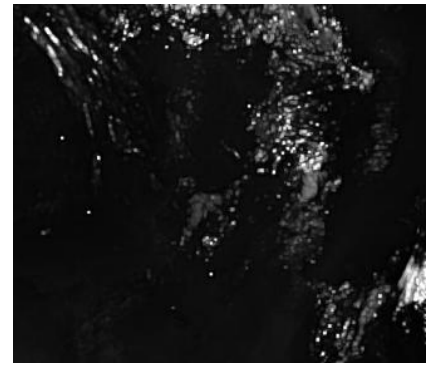

(b)

Fig. 2. Images obtained by the algorithm results in image. (a) Artwork. (b) Local contrast image

Fig. 2. (a) for the infrared image containing the target, and Fig. 2. (b) for the image after local contrast algorithm processing. It can be seen from the image that the image SNR is enhanced and the background is suppressed. The following table shows the SNR comparison between the local contrast map and the original image which are calculated by the LCM algorithm:

Table 1 The comparison of the original image and the saliency map target SNR

\begin{tabular}{ccc}
\hline taget & Artwork & Local contrast image \\
\hline taget 1 & 6.5321 & 8.5692 \\
taget 2 & 4.3015 & 6.3596 \\
taget 3 & 6.3205 & 9.1205 \\
taget 4 & 5.2584 & 5.8629 \\
taget 5 & 4.6258 & 5.4581 \\
\hline
\end{tabular}

But in the saliency map, there are a lot of false alarm targets in the cloud edge after local contrast algorithm, the gray value is very high, and the difficulty of the target segmentation is increased.

\section{Conclusion}

In this paper, the background suppression algorithm based on LCM algorithm has a large number of false alarm problems, it is proposed to eliminate the false alarm algorithm of cloud edge by combining the edge detection algorithm with Canny operator. By this algorithm, the background clutter can be suppressed, and the false alarm is eliminated, so that the target can be separated. After simulation experiment, we can see that the proposed algorithm can suppress the complex background and improve the image SNR.

\section{References}

[1] Chen C L P, Li H, Wei Y, et al.. A local contrast method for small infrared target detection[J]. IEEE Transactions on Geoscience and Remote Sensing, 2014, 52(1): 574-581.

[2] Zhang Libao, Zhang Jue. A new adaptive fusion method based on saliency analysis for remote sensing images $[\mathrm{J}]$. Chinese J Lasers, 2015, 42(1): 0114001.

[3] Canny J. A computational approach to edge detection [ J] . IEEE Transactions on Pattern Analysis and Machine Intelligence, 1986, 8(6): 679- 698.

[4] I.S.Reed,R.M.Gagliardi,L.B.Stootts.Optical moving target detection with 3D matched filtering[J].IEEE Trans AES.1988,24(4):327-336. 